\title{
Réponses de Claude Simon à quelques questions écrites de Ludovic Janvier
}

Ludovic Janvier et Claude Simon

\section{(2) OpenEdition \\ 1 Journals}

Édition électronique

URL : https://journals.openedition.org/ccs/890

DOI : $10.4000 /$ ccs. 890

ISSN : 2558-782X

Éditeur :

Presses universitaires de Rennes, Association des lecteurs de Claude Simon

Édition imprimée

Date de publication : 31 mai 2014

Pagination : 9-20

ISBN : 9782753533387

ISSN : $1774-9425$

Référence électronique

Ludovic Janvier et Claude Simon, « Réponses de Claude Simon à quelques questions écrites de Ludovic Janvier », Cahiers Claude Simon [En ligne], 9 | 2014, mis en ligne le 22 septembre 2017, consulté le 21 septembre 2021. URL : http://journals.openedition.org/ccs/890 ; DOI : https://doi.org/ $10.4000 / \operatorname{ccs} .890$ 


\section{RÉPONSES DE CLAUDE SIMON À QUELQUES QUESTIONS ÉCRITES DE LUDOVIC JANVIER}

Ludovic Janvier: - Vous avez écrit douze livres. Longue affaire. Quel rapport avez-vous avec ce discours entretenu depuis trente ans, et qui paraît au-dehors sous le nom de Claude Simon?

Claude Simon: - Longue affaire, comme vous dites. Et naturellement, en trente ans le rapport s'est quelque peu modifié. Mais il faudrait plutôt parler de rapports, au pluriel, car il y a, d'une part, ceux qu'entretient un écrivain avec son œuvre faite, de l'autre, ceux qu'il entretient avec l'œuvre qu'il est en train de faire.

Vous dites: "ce discours qui parait au dehors sous le nom de Claude Simon ». Pour moi, ce Claude Simon est un étranger, un autre? D'abord, et de toute façon, parce que, comme me le faisait un jour remarquer Maurice Merleau Ponty, le Claude Simon travaillant (travaillant son langage) n'est pas celui de la vie quotidienne, mais ce personnage que nous suscitons par notre labeur et qui se retire de nous dès que nous nous levons de notre table. Et puis, s'il est aussi bien évident que je ne suis plus le même homme qui a publié il y a cinq, dix ou vingt ans des romans sous la signature de Claude Simon (les cellules, les tissus qui me composent se sont renouvelés, certains sont définitivement morts), l'inévitable transformation se poursuit aussi pendant le temps passé à écrire chaque livre (le travail fourni contribuant même à l'accélérer). Et cela est si vrai qu'après avoir écrit les dernières pages je suis souvent amené à réécrire les premières produites par quelqu'un que je ne suis déjà plus...

En ce qui concerne mes rapports avec l'œuvre pendant que je l'écris (ou l'écrivais), si l'on entend par là mes motivations, elles aussi ont naturellement beaucoup changé au cours de toutes ces années. En gros, si on les énumère en leur attribuant un ordre de priorité, soit par exemple: 1) Écrire par besoin de faire; 2) Écrire pour représenter ; 3) Écrire pour communiquer ; 4) Écrire pour trouver, découvrir, je dirais que, parti de l'ordre, 1, 2, 3, 4 il y a trente ans, 
j'en suis peu à peu arrivé à l'ordre 1, 4, 3, le n 2 m'apparaissant de plus en plus douteux.

Ce que je veux dire, c'est que si la motivation profonde reste toujours la même (je crois qu'on écrit avant tout pour écrire - cet étrange plaisir solitaire -, comme un peintre peint avant tout pour disposer des couleurs sur une surface), j'ai lentement fini par me rendre compte (comme vous le voyez il m’a fallu du temps!) que l'écriture ne permettait pas de représenter ce que l'on appelle la réalité, mais au contraire de dire quelque chose qui entretient avec la « réalité » à peu près le même genre de rapports qu'une pomme figurée dans un tableau (c'est-à-dire constituée d'une infime couche de couleur étendue sur une toile) avec une pomme que l'on peut saisir et croquer.

\section{J. - Deux époques au moins dans votre travail. Du Tricheur au Sacre du} Printemps, c'est la première. La seconde commence avec Le Vent. Comment ces deux époques s'articulent-elles dans votre pensée? Ou la coupure formelle qui les sépare vous semble-t-elle totale? Par exemple, La Corde raide, plus que les autres textes de cette première période, annonce ou préfgure La Route des Flandres, Le Palace, Histoire. Que pensez-vous aujourd'hui de ce texte?

C. S. - Je ne ferais pas exactement la même séparation que vous. Du Tricheur au Vent y compris (mis à part le hiatus que constitue Gulliver: désorienté par les critiques qui avaient accueilli Le Tricheur, peu sûr de moi, j'ai cherché alors à prouver - entreprise absurde! - que je pouvais écrire un roman de facture traditionnelle. Excellente et fertile erreur au demeurant. Le résultat était édifiant: je ne pouvais pas! C'est alors que j'ai commencéà réfléchir sur les raisons de cette impossibilité), il n'y a pas à proprement parler de coupure nette, mais plutôt une lente évolution par tâtonnements. Avec L'Herbe, par contre, il me semble que quelque chose d'assez différent s'est produit. Un tournant était pris. Mais c'est seulement en écrivant Histoire que j'ai commencé à avoir une conscience plus nette des pouvoirs et de la dynamique interne de l'écriture et à me laisser guider plus par ce que l'écriture disait - ou " découvrait » - que par ce que je voulais lui faire dire ou " recouvrir ». Quant à la dernière partie de Pharsale (autre tournant) elle résulte de ce que j'avais enfin compris que l'on n'écrit - ou ne dit - jamais que ce qui se passe au présent de l'écriture (voir Stendhal dans Henri Brulard, lorsqu'il s'aperçoit, essayant de raconter son passage du Grand Saint-Bernard avec l'armée napoléonienne, qu'il est en train non pas de raconter ses souvenirs "véridiques " mais de décrire une gravure représentant cet événement qu'il a vu depuis et qui, comme il le dit, "a pris la place de la réalité »).

D'une certaine façon, bien sûr, La Corde Raide annonce La Route des Flandres, Le Palace, Histoire et même Pharsale, mais plutôt à la façon d'un répertoire, d'un inventaire des thèmes (je dis bien thèmes et non pas sujets) 
dans lequel j'ai ensuite puisé. Quant à ce que je pense de ce texte en soi, voici: il m'agace par un ton d'assurance et de provocation qui tient aux circonstances dans lesquelles il a été écrit et à l'âge que j'avais alors. Lorsque l'on est jeune, on n'est pas très sûr de soi ni des choses, et l'on éprouve le besoin de se rassurer en affirmant. Plus tard on est encore moins sûr, mais on a appris à endurer cette incertitude et à l'assumer.

L. J. - À partir du Vent, de quelle signification affectez-vous chaque moment, je veux dire chaque livre, de cette évolution qui mène jusqu'aux Corps conducteurs? (Le parcours représenté par chaque récit est de plus en plus réduit, le sujet paraît s'effacer d'un texte qui se fait, nettement dans vos deux derniers romans, constellation anonyme de figures, le foisonnement et la continuité de l'écriture narrative font place à une discontinuité, parfois à une fragmentation remarquables, etc.)

C. S - Il serait bien long de commenter étape par étape, c'est-à-dire roman par roman, l'évolution qui m'a mené jusqu'à celui que je viens de terminer. Mais assez vite (et dans Le Vent j'ai expressément formulé cela dans certains passages) j'ai été frappé par l'opposition, l'incompatibilité même, qu'il y a entre la discontinuité du monde perçu et la continuité de l'écriture.

Lorsque vous dites: "la continuité de l'écriture narrative fait place à une discontinuité ", est ce qu'il n'y a pas un malentendu sur le mot narrative? Parce que (Pardonnez-moi de vous contredire - mais peut-être n'est ce qu'une question de terminologie qui nous sépare?), il me semble, tout au contraire, que la continuité de mon écriture se fait de plus en plus rigoureuse. Alors, sans doute, faudrait-il s'entendre et chercher à voir quels sont, en fait, les pouvoirs de l'écriture romanesque, ce qu'elle peut narrer.

Bien sûr, dans les romans que j'appellerais non pas traditionnels (comme l'a dit Harold Rosenberg, la tradition en art c'est " la tradition du nouveau ") mais plutôt conventionnels (et non pas "balzaciens", comme le font abusivement certains en oubliant que les formes romanesques de Balzac étaient: a) absolument neuves et propres à Balzac et b) étroitement liées à un moment très précis de l'histoire dont nous sommes loin)... dans ces sortes de romans, donc, l'écriture prétend " narrer " ou " raconter " les aventures d'un ou plusieurs personnages " comme cela se passe dans la vie ", c'est-à-dire se succédant de façon linéaire dans le temps des horloges. Cette écriture narrative feint (si l'on y regarde de près on s'aperçoit en effet qu' elle ne peut que feindre) de se calquer sur la continuité et le cheminement de ce temps que l'auteur est censé suivre pas à pas. Et je dis censé parce que, en fait, même, dans le plus plat de ces sortes de romans, on assiste à une série d'accélérations ou de décélérations parfaitement « irréalistes ». Mais en fin de compte et en dépit de ces quelques 
concessions, la succession d'images, de descriptions d'objets de lieux, de personnages ou d'événements) que l'on nous propose, se fait là dans une sorte de cacophonie scripturale, et sans le moindre souci d'une véritable continuité de l'écriture. Il ne peut d'ailleurs en être autrement puisque ce ne sont pas tant les impératifs de l'écriture qui commandent, mais que ceux-ci "s'effacent" (comme on dit) derrière l'obligation de narrer une suite d'événements qui ne peut avoir la valeur exemplaire ou attractive (suspense) recherchée que par le mécanisme psychique ou social que met en lumière la façon dont ces événements, s'enclenchent, découlent les uns les autres dans le temps des horloges.

Passons sur cette valeur exemplaire ou probatoire d'une suite d'événements fictifs, nés du cerveau d'un auteur et que celui-ci combine à sa seule fantaisie, passons aussi sur le fameux et incroyable cliché du romancier qui, tout à coup, n'est plus " maître de ses personnages ", ou encore de celui (on a pu lire cette déclaration dans une récente enquête) qui prétend écrire "sous la dictée des masses "...

Plus d'un demi-siècle après Joyce, Kafka et Proust, ces pantalonnades semblent à peine croyables. Et pourtant, c'est encore d'après ce genre de critères que la quasi-totalité des critiques et du grand public continuent à juger de la valeur d'un roman.

En fait, tout peut se ramener, à une formule qui a fait ses preuves et qui est celle-ci: le dénouement de l'histoire racontée doit en être le "couronnement logique " (Henri Martineau). Et quelle logique? Eh bien, c'est très simple: tandis que, par exemple, le même Henri Martineau trouve d'une justesse psychologique admirable le coup de pistolet tiré par Julien Sorel sur $\mathrm{M}^{\mathrm{me}}$ de Rénal («ce retour d'adoration sentimentale envers sa victime»), Émile Faguet trouve, lui, ce même dénouement "plus faux qu'il n'est permis", et en conclut que Stendhal n'était pas intelligent. En somme, ces deux célèbres spécialistes discutent gravement des mérites d'une œuvre littéraire (et qui plus est d'une fiction, puisqu'il s'agit d'un roman) en trouvant moyen de ne parler à aucun moment du texte lui-même, comme deux psychologues ou sociologues (qu'ils ne sont pas) discuteraient d'un fait divers. Cela rappelle les fameuses descriptions, dans leur pur esprit " réalisme-socialiste " que Diderot faisait des compositions intimistes de Greuze. Vous allez me dire: "Martineau et Faguet! On en est tout de même plus là!...» Mais ouvrez donc aujourd'hui n'importe quel journal (ou même, hélas, certains périodiques dits littéraires), et voyez si ce ne sont pas là, obstinément, les références qui continuent à présider aux jugements des courriéristes: l'anecdote (que dans la première partie de ces « critiques » - la plus longue - on résume pour le lecteur, avant de s'attaquer, dans la deuxième partie - généralement un court appendice - au "style») est une "histoire d'une criante vérité ", "riche d'enseignements ", " révéla- 
trice des mœurs d'un certain milieu ou d'une certaine époque ", etc., ou, au contraire, "tirée par les cheveux ", témoignage "suspect ", "fantaisiste ", " partial », etc. Et quant à l'écriture, elle est, dans les dernières lignes, jugée (" plume alerte ", " récit vivement mené ", ou, au contraire, " lourde ", " laborieuse ", "récit qui se traîne ", etc.) par référence à des archétypes (en France Stendhal ou Flaubert généralement - quand ce ne sont pas Anatole France, Paul Bourget ou Montherlant) fixés une fois pour toutes.

Je ne crois pas caricaturer. L'explication de cette attitude en présence des romans, c'est que Martineau et Faguet (et leurs innombrables épigones) considèrent Julien Sorel et autres " héros ", comme des personnes "réelles". Pourtant Le Rouge et le Noir est un roman, donc, par définition, une fiction. Mais puisqu'il s'agit de fictions, qu'est-ce que l'écriture nous narre? Il ne s'agit pas de comptes rendus d'événements qui se sont effectivement produits et comme nous pouvons en lire dans les journaux, mais (quoique l'idée de Le Rouge et le Noir soit venue à Stendhal à la lecture d'un fait divers) d'aventures, d'événements, de personnages qui n'ont d'autre " réalité » que celle dans laquelle l'écriture les instaure, n'ont d'existence que par elle. Alors, dans ces conditions, comment diable (ainsi qu'on essaie souvent de nous le faire croire, ou même comme on nous le conseille), par quel bizarre tour de force, l'écriture pourrait-elle « s'effacer » derrière un récit et des événements qui n'existent que par elles? En fait, ce que l'écriture nous narre, ce sont sa propre aventure et ses sortilèges. Et si cette aventure est nulle, si ces sortilèges ne jouent pas, alors, le roman, quelles que puissent être par ailleurs ses prétentions didactiques ou morales, est lui aussi, tout simplement, nul.

Prenons l'exemple, d'un tableau où les passages, les relations entre les divers éléments (d'une robe à une tenture, de cette tenture à un mur, de ce mur au ciel sur lequel s'ouvre la fenêtre qui y est percée, etc. n'obéissent pas à des impératifs picturaux [accords ou dissonances savantes de couleurs, rythme, équilibre entre les masses colorées, etc.]), mais où n'importe quel vert voisine avec n'importe quel rouge, telle ligne se trouve à n'importe quelle distance de telle autre, etc., qui n'a autrement dit, aucune crédibilité picturale, eh bien, même si la scène qu'il "représente " est crédible, que ce soit une femme à sa toilette, des joueurs de cartes ou Lénine à tel congrès des Soviets, il ne retiendra même pas notre attention en tant que tableau et il est juste bon pour la poubelle, alors que tel autre, même représentant - ou plutôt: présentant-des scènes ou des personnages incrédibles (fantasmagories de Bosch, personnages à deux yeux pour un profil de Picasso, pêche à la baleine de Klee) aura, par la seule vertu de sa perfection picturale, une crédibilité, une présence.

Et il se passe exactement la même chose pour un texte où les divers éléments doivent avant tout s'organiser, se succéder (puisque l'écriture est par essence 
linéaire) pour des raisons impérieuses de qualité: associations ou contrastes d'images, cadences, parenté d'une ou plusieurs composantes des divers éléments (la madeleine de Proust, c'est en somme comme ce que l'on apprend maintenant aux enfants des petites classes lorsqu'on les initie à ce qu'en mathématiques on appelle les ensembles, c'est-à-dire, sommairement, que d'un groupe de triangles, dont un bleu, on passe à un autre groupe, composé, lui, de carrés, parce que l'un de ces carrés est bleu, puis de ce nouveau groupe à un groupe de cercles parce que l'un des carrés du deuxième groupe et l'un des cercles du troisième sont tous deux rouges, etc.). Par contre, si la seule règle qui préside à cette succession des éléments, c’est seulement cette nécessité « vériste ", dont nous avons parlé, de suivre dans le temps des horloges le déroulement d'une action (un personnage se trouve par exemple dans sa chambre, puis descend l'escalier, puis entame une discussion avec sa concierge, puis sort dans la rue, puis rencontre un autre personnage, etc.), l'écriture, alors ne narre finalement rien du tout puisque, nous le savons, cette chambre, ce premier personnage, cette concierge, ce second personnage qui n'ont pas d'existence " réelle " (on prétendra peut-être qu'ils sont " copiés d'après nature " - nous pourrons, si vous le voulez, revenir sur cette expression plus qu'abusive), n'ont alors même pas d'existence formelle.

Ma réponse à votre question a peut-être été un peu longue, mais cette question soulevait un monde de problèmes dans un domaine où la confusion semble entretenue à plaisir. Ce que j'ai voulu dire, c'est qu'à partir du moment où l'on n'écrit plus une histoire à prétentions exemplaires comme dans le roman conventionnel où l'intrigue est toujours plus ou moins au service d'une démonstration quelconque (le fameux " tableau de mœurs » ou la non moins fameuse "analyse psychologique »), l'écriture, alors, et ses nécessités propres prennent de plus en plus d'importance, et que si un ou plusieurs des éléments ou des événements thématiques peuvent en effet se trouver fragmentés, « oubliés » même par moments (en fait ils ne le sont jamais, existant toujours en filigrane), puis repris, puis "oubliés " de nouveau, par contre le texte, lui, va présenter une bien plus grande continuité puisque ses articulations, ses charnières, sa progression, ne dépendront plus que des relations qualitatives entre les éléments qui le constituent. Et je dirais que ce que ce texte narre alors sans plus de faux semblants, c'est cette continuité même, la façon dont il se construit peu à peu, cette progression qu'il guide lui-même: comme je l'ai indiqué plus haut, le sujet du roman, c'est cela. D'où le titre Histoire que j'ai donné à l'un de mes romans où certains critiques ont trouvé que "l'histoire " était plutôt "mince ", ou celui de Chronologie des événements pour la dernière partie de La Bataille de Pharsale où si, en effet, la chronologie selon le temps des horloges est bouleversée, par contre, l'ordre dans lequel se succèdent les 
événements écrits est le résultat d'un agencement rigoureux, la seule chronologie étant donc celle selon laquelle ils se succèdent dans le texte.

\section{J. - Vous avez souvent déclaré ne pouvoir rien inventer. Les mots redoublent-} ils l'histoire déjà vécue? Ont-ils pour fonction de la sauver, de lui offrir un terme, de la déplacer?

C. S. - J'ai dû dire ça dans un certain contexte, pour certains thèmes qui se trouvent dans mes livres, comme la guerre ou la révolution, thèmes qui sont liés à des situations vécues. Cela ne signifie pas que ces situations sont "représentées » dans mes livres ou que j'en témoigne. En fait, j'entendais par là que je ne pouvais écrire qu'à partir de mon expérience personnelle du monde. Mais est-ce qu'il n'en est pas ainsi pour nous tous? Il est bien évident, par exemple, que pour « inventer » le fameux tapis volant des Mille et Une Nuits il a bien fallu que le conteur oriental ait vu, au moins une fois dans sa vie, d'une part un tapis, d'autre part des objets volants (oiseaux ou feuilles emportées par le vent) ou, à défaut, qu'il en ait lu une description ou en ait contemplé une image.

Cela dit, il me semble que si l'on réfléchit à tout ce qui sépare et différencie l'objet ou l'événement " réel » de l'objet ou de l'événement écrit, du fait: 1) des imperfections de nos facultés de perception; 2) des imperfections de notre mémoire; 3) du choix, volontaire ou non de certaines de ses caractéristiques aux dépens d'autres qui sont rejetées ou passées sous silence; 4) de la nature même de l'écriture qui se déroule dans une durée, est donc obligée de dire successivement, ce qui, bien souvent, est perçu simultanément (d'où l'obligation encore de choisir un certain ordre, lui aussi fatalement arbitraire et subjectif); 5) des nécessités et contraintes formelles de l'écriture (syntaxe, composition, rythme, sons) ; 6) de la dynamique de celle-ci (nous sommes pour le moins autant conduits par notre langage que nous le conduisons)... eh bien, pour peu qu'on veuille se donner la peine de considérer cette effarante série de déformations, il devient alors bien évident que l'écriture ne peut prétendre, pour reprendre les termes dont vous vous servez, ni à "redoubler " l'histoire déjà vécue, ni à la "sauver ", ni à lui " offrir un terme ", mais à dire une histoire qui, encore une fois, n'entretient avec l'histoire "déjà vécue " que les très relatifs rapports de la pomme peinte avec la pomme "réelle ». (Mais peut-être est-ce que là ce que vous entendez par le terme " déplacer "? Chklovski définit le fait littéraire par: "le transfert d'un objet de sa perception habituelle dans la sphère d'une nouvelle perception " - par ailleurs, métaphore vient, comme vous le savez, du grec $\mu \varepsilon \tau \alpha \varphi \rho \rho \alpha ́$ qui signifie transport.

L. J. - Vous écrivez contre la mort, et vous la redites avec fascination. Vos "narrateurs " sont fascinés par la mort des autres, fascinés par leur mort, dont 
ils guettent dans les autres le travail, et dans le monde. L'écriture en vous a-t-elle lieu pour répéter cette fascination, etlou vous en délivrer? Ces lieux de pourrissements et d'à vau-l'eau, dont Barcelone est dans votre auvre le prototype, ces lentes batailles, l'érotisme comme désespéré, la nostalgie du corps portée par le concret de votre écriture, tout cela n'est-il pas répétition du travail de mort?

C.S. - Vous me posez là une question à laquelle vous êtes mieux qualifié que moi pour répondre. Écrire, c'est à la fois découvrir et se découvrir, et il est probable qu'un philosophe, un sociologue ou un psychanalyste, s'ils me lisent, en apprendront plus long que je n'en sais moi-même sur mon psychisme, mes refoulements, mes terreurs, mes obsessions, etc.

Sans doute peut-on dire, en effet, que la mort, le pourrissement, l'érotisme, la nostalgie des corps sont des thèmes qui reviennent dans mes romans. Ils sont aussi les thèmes de beaucoup d'autres, géniaux ou exécrables selon la façon dont ceux-ci sont écrits. Mais ce ne sont pas mes sujets, qui, comme je l'ai dit, sont précisément la façon dont mes divers romans sont écrits.

L. J. - On lit, en épigraphe d'Histoire, ces vers de Rilke: "Cela nous submerge. Nous l'organisons. Cela tombe en morceaux. Nous l'organisons de nouveau et tombons nous-mêmes en morceaux". Votre écriture mime le désordre et l'effondrement. Mais elle est un ordre. Si elle parle (il serait plus juste de dire: parlait, car ce mouvement culmine peut-être avec La Route des Flandres et Le Palace) de ce qui se défait, elle-même est un travail de constitution, bref, le livre est cette totalité "solide " qui énonce, donc domine le flux et le tâtonnement. Serez-vous d'accord si on interprète votre mise en mots comme le sauvetage acharné, la réponse acharnée à la perte subie dans et par l'histoire?

C. S. - Nous revenons à la question précédente. Si dans mes romans, je m'abstiens, pour tant que je peux, de faire de l'analyse psychologique ou sociologique ou encore de la métaphysique (il me semble qu'il vaut mieux laisser cela aux psychologues ou aux sociologues et aux philosophes), par contre, lorsque plus ou moins consciemment j'opte pour telle association plutôt que pour telle autre, je retiens telle caractéristique d'un objet plutôt que telle autre, décide de telle ou telle " mise en ordre ", eh bien, il ne fait aucun doute que ces différents choix, ces différentes options, me sont dictés par des impératifs qui, si je les discerne moi-même malaisément, découlent de la vie que j’ai menée, de la société et du milieu dans lesquels j'ai vécu, des événements historiques auxquels j'ai été mêlé, de même que de ma libido, mon éducation, etc.

Vous dites: "le sauvetage acharné, la réponse acharnée à la perte subie et dans et par l'histoire". Il me semble que l'on ne sauve jamais rien de ce qui est perdu. Même un objet matériel retrouvé n'est plus le même du fait que, pendant un temps, il a été perdu. Vous connaissez le fameux apologue des 
neuf pièces imaginé par Borges dans la « fiction » intitulée Tlon, Uqbar, Orbis Tertius (qui définit peut-être - avec toutes les habiles précautions d'ambiguïté que peut prendre Borges - un "art poétique »), apologue réfuté par les habitants de Tlon qui nient l'identité des pièces retrouvées et des pièces perdues. La recherche de Proust ne l'a pas conduit à retrouver le temps, mais à produire un objet écrit qui a sa propre temporalité. Je crois que pas plus qu'il ne peut être témoignage (sauf indirectement et pas par ce qui le constitue en tant qu'art), l'art ne peut être non plus une entreprise de récupération.

\section{J. - La peinture n'est jamais absente de votre texte, ce que confirme Orion} aveugle. On dirait qu'elle représente pour vous l'art le plus libéré de ce qu'on nomme le réel, donnant ainsi une leçon à la littérature. D'autre part, quand on connaît la pratique du collage que vous développez en marge de votre cuvre écrite, et quand on vous entend dire que vous auriez soubaité être peintre, la question simpose: quels rapports (votre texte et vous) entretenez-vous avec l'ouvre peinte?

C. S. - Comme vous l'avez vu, je me suis souvent aidé, pour vous répondre, de comparaisons avec la peinture. Peut-on dire cependant, qu'elle soit l'art le plus libéré de ce que l'on nomme le réel, surtout quand on la compare à la littérature? Cela supposerait qu'il existe une écriture réaliste ou vériste. Il me paraît que la série des déformations successives que j'ai énumérées tout à l'heure et qui différencient l'écrit du perçu (ne parlons même pas du " réel »!...) montre assez bien qu'il ne peut y avoir de littérature "réaliste ", même si elle se veut et se proclame telle.

Peut-être la peinture permet-elle plus facilement que la littérature (entachée de la supercherie naturaliste) de se rendre mieux compte de la vanité de toute prétention au réalisme. Pour le grand public il est certain qu'est considérée comme "réaliste " toute œuvre d'art dont l'aspect "ressemble " non pas à la nature (un même objet a mille visages), mais à l'image de la nature à laquelle il est accoutumé depuis longtemps. On connaît la boutade: "La nature imite l'art "... Habitué aux fruits de Chardin, le public trouvait les fruits peints par Cézanne non ressemblants (non réalistes), habitué ensuite aux pommes de Cézanne, il a trouvé les pommes de Picasso agressivement irréelles (on se foutait du spectateur!), habitué plus tard aux pommes de Picasso..., etc.

Eh bien, en se gardant d'analogies dangereuses (l'écriture n'est pas la peinture, le pouvoir évocateur de la figuration picturale d'un corps est tout autre que celui de la description scripturale d'un corps, la peinture est surface, simultanéité, l'écriture est linéarité, durée, etc.), on peut, cependant, parce que dans les deux cas il s'agit d'art, trouver certains points communs entre écriture romanesque et peinture. Et puisque vous me parlez de mes collages (pour lesquels je me sers d'éléments préfabriqués: des personnages, des animaux, des 
fleurs, etc. découpés dans des magazines ou des reproductions de tableaux), j'ai appris en les faisant, plusieurs petites choses qui, je crois, sont aussi valables pour mes romans, et surtout celle-ci: c'est que si un élément (disons, par exemple, un cheval noir) commande ou attire auprès de lui d'autres éléments à la fois par ce que l'on pourrait appeler la morphologie du signe en soi (noir) et par son signifié (cheval), il faut toujours sacrifier le signifié aux nécessités plastiques, ou, si l'on préfere, formelles, c'est-à-dire qu'avant toute autre considération il faut que le noir (et l'arabesque du dessin) s'accorde (harmonie ou dissonance) avec la ou les couleurs (et les arabesques) des éléments avec lesquels il va voisiner sans se demander ce que peut (par exemple) bien faire un cheval dans une chambre à coucher ou encore à côté d'un pope en chasuble plutôt que galopant au bord de la mer ou dans une prairie.

Bien sûr dans le roman, à la différence des collages, on ne doit jouer qu'avec un nombre limité de thèmes (sans quoi ce serait alors la tentative décevante de l'écriture automatique des surréalistes qui ouvrait des successions de parenthèses sans jamais les fermer). Mais la leçon, à mon avis d'une importance primordiale, c'est que si, sans se préoccuper de leurs signifiés, on réussit à établir entre deux signifiants un rapport formel " parlant", il se produit alors un phénomène qui semble tenir du prodige : à savoir que va apparaître de surcroît (en "prime", pourrait-on dire) une ouverture signifiante, un sens ambigu, incertain, "tremblé " comme dirait Barthes, non explicité, mais souvent plus riche et générateur (ou chargé) de vibrations que celui que l'on aurait pu établir entre deux éléments choisis seulement en fonction de leurs signifiés (cheval-plage ou cheval-prairie) et dont la manipulation a montré, en dépit du rapport apparent de ces derniers, l'incompatibilité formelle.

Il va naturellement sans dire que cette loi (accord et dépendance entre les morphologies des signifiants) n'empêche pas les rapprochements de signifiants en fonction de leurs signifiés, mais ceux-ci ne peuvent jamais avoir de crédibilité picturale ou scripturale si la loi en question n'est pas observée et, par voie de conséquence, toute combinaison, pour si séduisante qu'elle puisse paraître au niveau des signifiés, doit être rejetée sans hésitation lorsqu'elle ne peut y satisfaire. Seulement, loin de constituer une gêne, un obstacle (comme le croient ceux qui s'écrient d'une façon assez comique: "Ah, si je pouvais arriver à dire ce que je veux dire! Mais les mots trahissent ma pensée!...»), les nécessités formelles sont au contraire, en elles-mêmes, créatrices. Comme me le disait Raoul Dufy un jour que je le regardais peindre: « Il faut savoir abandonner le tableau que l'on voulait faire au profit de celui qui se fait. " 
L. J. - Pouvez-vous, depuis l'après-coup où vous met la lecture de vous-même, apercevoir la fonction ou la valeur d'usage de votre cuvre dans l'histoire des hommes où elle prend place? D'autre part, lui souhaitez-vous, lui voulez-vous un rôle?

C. S. - Non, il m'est impossible d'apprécier la fonction ou la valeur d'usage de mon ouvre dans l'œuvre dans l'histoire des hommes. Vous savez bien, comme moi, qu'écrire c'est une sorte de travail de taupe et que l'écrivain se trouve à peu près dans la situation d'un artisan qui ferait des ouvrages de cuivre repoussé (ou martellé) sans pouvoir regarder le côté "face " de son œuvre. Je ne peux donc que me contenter de souhaiter que la mienne prenne place dans l'histoire, et elle n'y prendra place que si elle relève de la dimension de l'art, c'est-à-dire dans la mesure où elle aura apporté des formes neuves participant de et à l'incessante transformation du monde.

L. J. - Quel sens a pour vous, aujourd'hui et depuis votre pratique de l'écriture, le mot réalisme?

C. S. - Je crois que j'ai déjà répondu plus haut à cette question.

L. J. - La littérature d'aujourd'hui: comment la voyez-vous? Le Nouveau Roman? Tel Quel? La confluence évidente (?) de la critique et de la fiction?

C. S. - Je ne la vois pas. J'essaie de la faire. C'est déjà suffisamment difficile. Quant à la critique (je veux dire une certaine critique) vous savez comme moi et même mieux que moi l'importance tout à fait nouvelle qu'elle a prise et tout ce que certains travaux récents ont appris sur leur art aux écrivains de fictions. Sur cette importance et sur la relation "critique-fiction " dont vous parlez, je crois qu'il est bon de rappeler la percutante formule de Jean Ricardou: «la lecture-qui-écrit ».

L. J. - Dans votre préface à Orion aveugle vous écrivez: "Je ne connais pour ma part d'autres sentiers de la création que ceux ouverts pas à pas, c'est-à-dire mot après mot, par le cheminement même de l'écriture. "D'un côté, votre projet et votre désir. De l'autre, l'aventure de ceux-ci dans le langage, puisque c'est le corps des mots qui leur donne une histoire. Comment est-ce, de s'engager dans ce travail, de sy maintenir, de découvrir mot après mot, de construire cette recherche, puis d'en finir avec elle?

C. S. - À cette phrase de la petite préface sue j'ai écrite pour Orion aveugle, quelqu'un m'a objecté: "Ce ne sont pas les mots qui vous conduisent: ce sont les images ". Vous répondez parfaitement à cette objection lorsque vous dites: "c'est le corps des mots qui leur donne une histoire» (et, j'ajouterais: un pouvoir). Parce qu'il est un fait: c'est que si je n'écris pas, c'est-à-dire si je ne commence pas par tracer des mots sur le papier, alors rien ne se produit. Pourquoi? 
Je serais bien incapable de le dire. Sans doute de plus savants que moi dans les problèmes de l'écriture pourraient-ils l'expliquer. Mais ce n'est jamais assis dans un fauteuil, ou couché sur mon lit, ou pensant à des "images " que d'autres viennent s'agglutiner à celles-ci, c'est seulement en écrivant.

Ce que toutefois il faut peut-être bien préciser, pour un certain public qui pourrait penser après tout ce que je viens de dire qu'en fin de compte, dans ces conditions, il ne s'agit plus que d'un " jeu avec les mots ", ou de " jeux de mots", c'est que, s'il y a en effet jeu avec les mots (aussi bien jeu pour le jeu, comme un enfant qui joue avec des cubes ou encore comme les jeux sportifs - faire passer un ballon entre deux poteaux en observant un certain nombre de règles convenues -, ou encore comme le mathématicien - de mon temps le cours de mathématiques supérieures commençait par le chapitre: Arrangements, Permutations, Combinaisons), ce qu'il ne faut absolument pas perdre de vue c'est ce rapport perpétuellement ambigu, qui existe entre les mots et les choses, ce jeu (dans le sens, cette fois, où l'on dit qu'une mécanique, une transmission a du « jeu ", c'est-à-dire qu'entre l'impulsion donnée et le mouvement produit s'interposent une série de décalages du fait que les différentes pièces ne sont pas étroitement emboitées ou articulées). Si, comme je l'ai écrit, on ne doit jamais oublier que le mot feu n'est pas le feu, que le mot sang n'est pas du sang, on ne doit pas oublier non plus que les mots feu et sang nous renvoient aux images et aux concepts du feu et du sang. Paul Valéry faisait très justement remarquer que la Joconde ou la Vénus de Milo n'avaient ni système nerveux ni foie. Néanmoins, elles ne sont pas seulement des taches de couleur sur une toile ou un bloc de marbre harmonieusement taillé. Si la célèbre métaphore de Saint Pol Roux mamelle de cristal produit un objet encore jamais vu et qui est autre chose qu'une carafe, et cela par la mise en rapport de deux substantifs dont non seulement le sens, mais encore la matière, la morphologie s'affrontent (mollesse de mamelle s'opposant à la dureté du mot cristal) établissant ainsi un rapport à la fois neuf et parlant, eh bien, ce nouvel objet n'aurait pourtant malgré cela aucune existence si ces deux mots rapprochés et leur contexte ne faisaient pas surgir dans notre esprit les images ou les concepts d'une mamelle, du cristal et d'une carafe.

Vous me demandez encore: "Comment est-ce de s'engager dans ce travail, de s'y maintenir, de découvrir mot après mot, de construire cette recherche...? " Je crois, avec tout ce que je viens de dire vous avoir répondu: c'est proprement fascinant. Susciter, contrôler, relancer ce jeu, ou si vous préférez cet extraordinaire processus qui fait que, parti avec un vague projet, on voit peu à peu se faire au fur et à mesure de l'écriture " autre chose " (non que cela se fasse tout seul, est-il besoin de le préciser?), et que cet "autre chose " soit finalement, du moins en ce qui me concerne, beaucoup mieux que le projet initial, cela n’a pas fini de m'émerveiller. 
Cet entretien, rappelons-le, figure dans le numéro consacré à Claude Simon de la revue Entretiens des éditions Subervie ${ }^{1}$, fondée par un petit noyau d'enthousiastes de la littérature et de la poésie contemporaines dont le poète Frédéric-Jacques Temple: il voisine avec une republication des "Cinq notes sur Claude Simon de Maurice Merleau-Ponty", des paroles de traducteurs ou de critiques étrangers (T. Bishop, J. Fletcher, C. G. Bjurström ou R. Howard), des points de vue novateurs d'essayistes ou de théoriciens de la littérature (Raymond Jean, Jean Ricardou, Serge Doubrovsky) et accueille également le texte bref encore intitulé Femmes, sur vingt-trois peintures de Joan Miró, qui deviendra La Chevelure de Bérénice ${ }^{2}$.

Il s'agit là d'un épitexte qui mérite d'être examiné avec attention. Les conditions dans lesquelles il a été réalisé font que le romancier a pris le temps de développer sa réflexion jusque dans ses ramifications les plus fines. On le sent en confiance face à un interlocuteur à l'écoute, particulièrement aigu dans sa progression. Rappelons que Ludovic janvier (né en 1934) est tout à la fois critique et écrivain, qu'au moment de l'échange, il est connu pour s'être attaché à expliciter les options esthétiques du Nouveau Roman et qu'il est l'auteur de deux livres essentiels sur Beckett, parus au Seuil ${ }^{3}$.

Cet échange s'oriente très vite vers un bilan de trente années de production textuelle. Simon vient de faire paraître Les Corps conducteurs (1971) qui est un peu l'acmé de sa période expérimentale. Nous avons donc la chance, en quelque sorte, de relire cet état des lieux d'une œuvre au début de la décennie 70, avec la connaissance que nous avons de l'œuvre ultérieure. Il est d'ailleurs stimulant de constater que le romancier s'installe d'emblée dans le relativisme d'une évaluation rétrospective, saisissant ici encore l'occasion de rappeler qu'il ne peut plus coïncider avec celui qui a publié cinq, dix ou vingt ans plus tôt.

1. Les autres membres fondateurs sont Denys-Paul Bouloc, qui fut l'ami d'Artaud lorsque celui-ci séjourna à l'asile de Rodez, Jean Digot co-fondateur avec Frédéric-Jacques Temple des « Journées de poésie » (à partir de 1951) et Pierre Loubière, poète-paysagiste, auteur entre autres recueils de Mémoire buissonnière (1976).

2. La première édition du texte, à tirage limité (sous le titre Femmes) est celle des éditions Maeght (1966), illustrée de reproductions de peintures de Joan Miro. Le texte est reparu sans illustrations en 1984 aux éditions de Minuit: il figure dans le premier volume des Euvres de Claude Simon dans la « Bibliothèque de la Pléiade ", dir. A. Duncan, avec la collaboration de J. Duffy, 2006.

3. Pour Samuel Beckett, Minuit, 1966, et Samuel Beckett par lui-même, Le Seuil, 1969. 
Dans cet ensemble de réponses, il est loisible de repérer un certain nombre d'affirmations que nous retrouverons différemment modulées dans les autres textes " théoriques" de l'auteur, souvent plus tardifs, entretiens divers, conférences données ici ou là pour une circonstance précise, essais importants comme la "Préface à "Orion aveugle" " (1970), "La Fiction mot à mot " (1972), ou le Discours de Stockholm (1986).

Quelles seraient ces grandes lignes récurrentes?

D'abord, une défiance toujours exprimée par rapport à une littérature de représentation, la promotion en contraste d'une littérature de recherche dans la lignée proustienne, le refus d'une causalité socio-psychologique soustendant une intrigue romanesque, l'allergie à un dénouement du récit présenté comme le " couronnement logique " d'un itinéraire de personnage, et le rappel également que cette défiance vaut d'être reformulée dans la mesure où la critique journalistique n'est malheureusement pas sortie d'un tel schéma, oubliant souvent que le chemin d'une création renouvelée a été indiqué par les œuvres de "Kafka, Joyce et Proust».

Ensuite, une redéfinition de la notion de « continuité » en contexte narratif. Manière pour Simon de souligner que le roman tel qu’il le conçoit, n’en déplaise à la critique académique, ne va pas dans tous les sens, qu'il substitue simplement à une linéarité factice, fondée sur une succession arbitraire d'épisodes fictifs, une continuité d'écriture née d'associations qualitatives entre divers éléments du récit. "Continuité » dont la peinture la plus audacieuse est également illustrative lorsqu'elle se soumet à des impératifs rigoureusement picturaux.

Enfin, une mise au point sur le sens de l'expression " ne pouvoir rien inventer » qui lui est rappelé par Ludovic Janvier comme étant sienne et qui, selon son dire, n'équivaut ni à un brevet de réalisme ni à une velléité de témoignage. Certes, le romancier concède que des situations éprouvées à Barcelone en 1936 ou pendant la guerre ont été déterminantes dans son parcours d'existence et qu'on ne peut écrire qu'à partir d'une expérience sensible mais il prend soin d'énumérer toutes les médiations déformantes par lesquelles passe " un événement réel " pour devenir "un événement écrit ", imputables aux approximations de la perception, à une mémoire plus ou moins faillible, au projet du scripteur, à l'élaboration d'une poétique ou même au respect des contraintes productrices de la langue.

Mériteraient d'être également soulignés deux points plus spécifiques à cet entretien.

Il y aurait, par exemple, le regard que Simon porte sur les débuts de son itinéraire de romancier: à Janvier qui dessine une première période s'étendant du Tricheur (1945) au Sacre du printemps (1954), et décerne au Vent (1957) le statut de texte fondateur, Simon répond en plaçant la barre de scansion avant L'Herbe 
(1958), préférant deviner dans ce qui précède, mis à part le faux-pas de Gulliver, " une lente évolution par tâtonnements ", et datant de la rédaction d'Histoire (1967), la prise de conscience d'une nouvelle voie essayée avec L'Herbe, le choix de s'abandonner en conscience "à ce que l'écriture découvr[e] ". Il accorde également à son interviewer que $\mathrm{La}$ Corde raide annonce bien $\mathrm{La}$ Route des Flandres (1960), Le Palace (1962) et Histoire mais "à la façon d'un répertoire, d'un inventaire de thèmes » et il a des mots un peu durs pour stigmatiser "le ton d'assurance et de provocation " de ce récit de jeunesse en lequel il décèle une forme d'immaturité.

Autre moment important de l'entretien, celui où Ludovic Janvier, que l'on sait également grand lecteur de Beckett, tente de conduire Claude Simon du côté d'une interrogation sur la possible fascination de la mort qui traverserait son œuvre. Simon ne se laisse assurément pas enfermer dans une question qui relève pour lui de la psychologie ou de la métaphysique. Néanmoins, il reconnaît que " le pourrissement, l'érotisme, la nostalgie du corps » sont effectivement des thèmes qui lui sont familiers. Et il entend ce que Janvier suggère en lui citant l'épigraphe d'Histoire, à savoir que ses textes pourraient être lus comme un combat contre le tragique de l'Histoire. Mais il refuse énergiquement le principe d'une tentative de salut par l'art. Tout au plus, accepte-t-il l'idée que son œuvre "prenne place dans l'histoire "; et elle ne le pourra, estime-t-il, que «dans la mesure où elle aura apporté des formes neuves participant de et à l'incessante transformation du monde ». Pas de position de surplomb donc mais une confiance réaffirmée dans le travail qui s’accomplit à travers la durée. "Vous savez bien $[. .$.$] qu'écrire, c'est une sorte de travail de taupe »...$ 